\title{
Clinical features, neuroimaging findings and treatment outcomes in non ketotic hyperglycemic hemichorea-hemiballismus
}

\author{
Raghunandan Nadigi, Sagar Badachi2, ${ }^{2,}$, G R K Sarma ${ }^{3}$, Sharath Kumar GG ${ }^{4}$, Thomas Mathew ${ }^{5}$ \\ ${ }^{1,4}$ Associate Professor, ${ }^{2}$ Assistant Professor, ${ }^{3,5}$ Professor, Dept. of Neurology, St. John Medical College, Bengaluru, Karnataka, \\ India
}

*Corresponding Author:

Email: sagar_badachi@yahoo.com

\begin{abstract}
Introduction: Nonketotic hyperglycemic hemichorea-hemiballism (NKHH) is an uncommon hyperkinetic movement disorder. It is the second most common etiology after stroke affecting basal ganglion. Only a few hundred cases are reported so far in literature.

Objectives: The study attempts to systematically highlight clinical profile and neuroimaging findings in 15 patients of Nonketotic hyperglycemic hemichorea-hemiballism.

Materials and Methods: Patients with hemichorea/ hemiballism associated with hyperglycemia were included in the study. The period of the study was from January 2008 to September 2018. Neurological examination, Demographic data, clinical, biochemical, and neuroimaging findings were captured from the medical records. Details of the involuntary hyperkinetic movements such as type and side(s) involved were noted. Treatment was aimed to control glycemic status and neuroleptic drugs. Descriptive statistics were used wherever necessary.

Results: During the study period, 52 patients had hemichorea/ hemiballismus.27 patients had stroke affecting basal ganglia.6 patients had granuloma affecting basal ganglia. 4 patients had Wilson's disease.15 patients had NKHH. Among 15 patients of NKHH, 9 patients were females and 6 were males. (Female to male 1.5:1). The mean age at the onset was 70.2 years (Range 58 85). The mean serum glucose levels measured after the onset of NKHH was $439.93 \mathrm{mg} / \mathrm{dL}$ (Range 342-532). The mean glycated haemoglobin was $10.98 \%$ (Range 9.1-18.5.Neuroimaging showed involvement of striatum in 13 patients.

Conclusion: NKHH is a rare hyperkinetic movement disorder. Elderly poorly controlled diabetics and female sex are most commonly affected. Neuroimaging findings are distinct. Further studies are needed to elucidate pathogenesis of this disease and for determining prognosticating factors.
\end{abstract}

Keywords: Non ketotic, hyperglycemia, Hemichorea-hemiballism, Putamen, Striatum.

\section{Introduction}

Hemichorea-hemiballism (HCHB) is an uncommon hyperkinetic movement disorder. It is characterized by continuous unilateral involuntary and non rhythmic movements of one or both limbs. ${ }^{1}$ The most common cause for hemichorea-hemiballism is stroke affecting basal ganglion. Non ketotic hyperglycaemic hemichorea-hemiballism (NKHH) is the second most common aetiology. Elderly, Asian population with poorly controlled Type 2 diabetics are most commonly affected. ${ }^{2}$ Patients of NKHH exhibit contralateral striatal abnormalities on CT/MRI brain. NKHH is a very rare disorder, only a few hundred cases are reported so far in the literature. There are only few case reports and case series of NKHH reported from India. To the best of our knowledge, this is the largest cohort of patients with NKHH from India.

\section{Objectives of the study}

1. To describe clinical profile of patients with NKHH.

2. To describe the MRI Brain imaging findings in patients with NKHH

3. To identify the prognostic factors in patients with $\mathrm{NKHH}$

\section{Materials and Methods}

The study was conducted at Department of Neurology; from a tertiary care university-affiliated Hospital from South India.

Study Population: Consecutive patients presenting with hemichorea/hemiballism associated with hyperglycaemia were included in the study.

Study design: Cross-sectional descriptive study

Period of study: January 2008 to September 2018

Demographic data, clinical, biochemical, and neuroimaging findings were captured from the medical records. Details of the involuntary hyperkinetic movements such as type and side(s) involved were noted. Patients with low-amplitude, random, flowing movements were considered as chorea and patients with proximal and large-amplitude movements were considered as ballism. Hyperglycemia was treated with insulin. Neuroleptic drugs were also administered.

Descriptive statistics were used. Continuous variable is expressed as mean $\pm \mathrm{SD}$ or median (Range) if non-normally distributed. Categorical data is expressed in proportions. 


\section{Results}

During the study period, 52 patients had presented with hemichorea/hemiballismus to our department. Hemichorea was present in 30 patients and 22 patients had presented with hemiballismus. Of these 27 patients were found to have stroke involving basal ganglia, 15 patients had NKHH, 6 patients had granuloma affecting basal ganglia and 4 patients had Wilson's disease. The Fifteen patients who had non ketotic hyperglycemia were included in the study for detail analysis.

Demography and Clinical presentation: Of the 15 patients with NKHH 9 patients were females and 6 were males (female to male ratio was 1.5:1). The mean age at the onset was 70.2 years(range 58 -85). All patients were right handed.4 patients had newly detected Type $2 \mathrm{DM}$. IN the remaining 11 patients with Type $2 \mathrm{DM}$, the mean duration of DM was 11.8 years (range 7-20 years). The mean duration of presentation to our centre after symptom onset was 6.13 days (range 2- 20 days). (Table 1) The abnormal movements noted in these patients were hemichorea in 5 patients and hemiballismus in 10 patients. In 10 patients $(67 \%)$ the abnormal movements were noticed on the left side of the body and in other 5 patients $(37 \%)$ on right side of the body. (Table 2)

Biochemical Parameters: The mean serum glucose levels measured after the onset of NKHH was 439.93 $\mathrm{mg} \%$ (range $342-532 \mathrm{mg} \%$ ). The mean glycated haemoglobin was 10.98 (range 9.1-18.5). Urine ketones, serum electrolytes, renal function tests and liver function tests were normal in all patients

Neuroimaging: MRI brain was done in all patients. 13 patients had contralateral striatal abnormalities and in 2 patients there were no changes noted in the contralateral basal ganglia. Among 13 patients of striatal involvement, putamen was involved in all patients and caudate nucleus was involved in only 9 patients. In these thirteen patients $\mathrm{T} 1 \mathrm{~W}$ images revealed unilateral striatal hyperintensity contralateral to the side of abnormal movements with mild hypo intensity on T2 FLAIR. DWI did not show any diffusion restriction. CT brain was done in only 8 patients and it showed mild hyper density in contralateral putamen. Representative images are depicted in figures 1,2, 3 .

Treatment: Insulin was administered to all patients for controlling hyperglycemia. $2.5 \mathrm{mg}$ of Haloperidol was also initiated and the dose was titrated based on the symptoms of the patients.

In the study it was noticed that, with the control of sugars and a small dose of haloperidol $2.5 \mathrm{mg}$ administration, involuntary movements subsided in 13 patients. The average duration for the involuntary movements to subside was 6.3 days (range 2- 12 days).

Only in two patients, chorea persisted even after 2 months from the symptom onset. These two patients had presented to us more than two weeks after the onset of chorea. Both of them had Right sided chorea and their MRI of brain did not reveal any abnormalities in the contralateral basal ganglion.

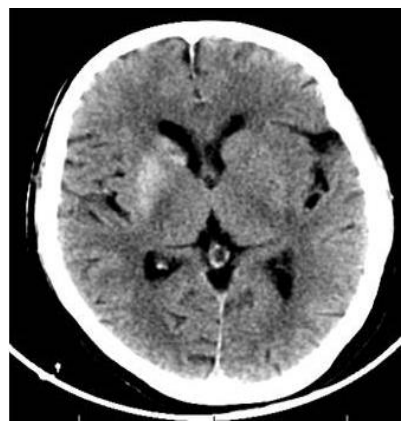

Fig. 1: CT brain showing hyperdensity of right

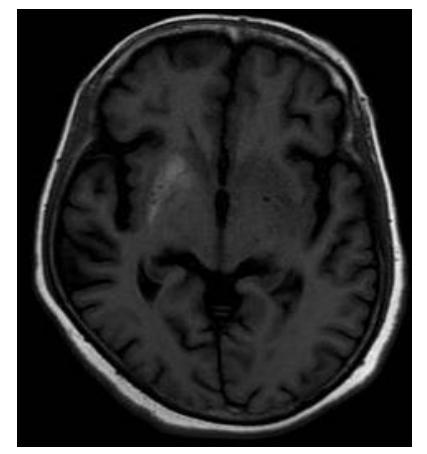

Fig. 2: Axial T1 image showing hyperintensity in right putamen

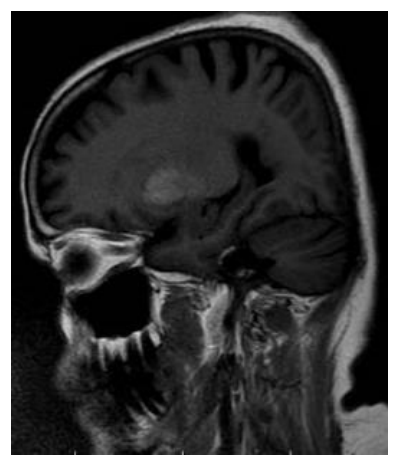

Fig. 3: MRI brain sagittal TI showing hyperintensity of right putamen

Table 1: Demographic data and Glycemic status of patients

\begin{tabular}{|c|c|c|c|c|c|c|c|}
\hline Age & Sex & Handedness & $\begin{array}{c}\text { Side of } \\
\text { body }\end{array}$ & $\begin{array}{c}\text { Duration } \\
\text { of DM }\end{array}$ & $\begin{array}{c}\text { Duration of } \\
\text { symptoms }\end{array}$ & $\begin{array}{c}\text { GRBS } \\
(\mathbf{m g} / \mathbf{d L})\end{array}$ & $\begin{array}{c}\text { HbA1c } \\
(\mathbf{\%})\end{array}$ \\
\hline 67 & $\mathrm{~F}$ & $\mathrm{R}$ & $\mathrm{R}$ & 12 years & 7 days & 456 & 11 \\
\hline 82 & $\mathrm{~F}$ & $\mathrm{R}$ & $\mathrm{R}$ & New & 2 days & 375 & 9.2 \\
\hline 85 & $\mathrm{M}$ & $\mathrm{R}$ & $\mathrm{L}$ & New & 3 days & 480 & 18.5 \\
\hline
\end{tabular}




\begin{tabular}{|c|c|c|c|c|c|c|c|}
65 & $\mathrm{~F}$ & $\mathrm{R}$ & $\mathrm{L}$ & 8 years & 15 days & 532 & 10 \\
\hline 58 & $\mathrm{~F}$ & $\mathrm{R}$ & $\mathrm{L}$ & 10 years & 2 days & 476 & 12 \\
\hline 61 & $\mathrm{~F}$ & $\mathrm{R}$ & $\mathrm{L}$ & New & 3 days & 504 & 14.3 \\
\hline 58 & $\mathrm{M}$ & $\mathrm{R}$ & $\mathrm{L}$ & New & 4 days & 475 & 9.5 \\
\hline 62 & $\mathrm{M}$ & $\mathrm{R}$ & $\mathrm{R}$ & 10 years & 15 days & 345 & 10.1 \\
\hline 65 & $\mathrm{M}$ & $\mathrm{R}$ & $\mathrm{R}$ & 12 years & 20 days & 456 & 12 \\
\hline 82 & $\mathrm{~F}$ & $\mathrm{R}$ & $\mathrm{L}$ & 9 years & 3 days & 516 & 10.2 \\
\hline 67 & $\mathrm{~F}$ & $\mathrm{R}$ & $\mathrm{L}$ & 7 years & 4 days & 342 & 9.6 \\
\hline 72 & $\mathrm{~F}$ & $\mathrm{R}$ & $\mathrm{L}$ & 11 years & 2 days & 388 & 9.1 \\
\hline 75 & $\mathrm{M}$ & $\mathrm{R}$ & $\mathrm{L}$ & 12 years & 3 days & 456 & 9.5 \\
\hline 70 & $\mathrm{M}$ & $\mathrm{R}$ & $\mathrm{L}$ & 20 years & 5 days & 432 & 9.6 \\
\hline 85 & $\mathrm{~F}$ & $\mathrm{R}$ & $\mathrm{R}$ & 12 years & 4 days & 366 & 10.2 \\
\hline
\end{tabular}

F-female, M- Male, R- Right, L- left

Table 2: Clinical presentation, neuroimaging findings and outcome

\begin{tabular}{|c|c|c|c|c|}
\hline Putmen & Caudate & HemiChorea & HemiBallismus & Improvement \\
\hline L & L & Y & N & 3 days \\
\hline L & L & Y & N & 4 days \\
\hline R & R & Y & N & 7 days \\
\hline R & R & Y & N & 4 days \\
\hline R & R & N & Y & 3 days \\
\hline R & N & N & Y & 6 days \\
\hline R & N & N & Y & 10 days \\
\hline N & N & N & Y & 2 months \\
\hline N & N & N & Y & 3 months \\
\hline R & R & N & Y & 12 days \\
\hline R & R & N & Y & 2 days \\
\hline R & R & N & Y & 4 days \\
\hline R & R & N & Y & 7 days \\
\hline R & N & N & Y & 12 days \\
\hline R & N & Y & N & 8 days \\
\hline
\end{tabular}

R- Right, L- left, Y- Yes, N-No

\section{Discussion}

Hemichorea-hemiballism is an uncommon hyperkinetic movement disorder. The disease is characterised by triad of unilateral involuntary movements, contralateral striatal abnormalities on neuroimaging and hyperglycemia in patients with known or previously unrecognized diabetes mellitus. It was first described by Bedwell in $1960 .{ }^{4}$ Only a few hundred cases are reported in literature so far. ${ }^{5}$ No epidemiological studies are available in literature. The estimated prevalence of $\mathrm{NKHH}$ is less than 1 in $100,000 .{ }^{6}$ Most of the patients of NKHH were of Asian origin; however cases have been published from all ethnic backgrounds.

In the present study, 9 patients were females and 6 were males. (Female to male ratio is 1.5:1). Earlier studies point towards female preponderance (female to male ratio is 1.8:1). ${ }^{2}$ Hypothesis for elderly female predilection for NKHH is that the declining oestrogen concentration in postmenopausal women results in hypersensitivity of nigrostriatal dopamine system receptors leading to enhancement of striatal dopamine system function. ${ }^{7}$
In the present study the mean age at the onset was 70.2 years. Earlier studies have shown that older age appears to be the greatest risk factor for NKHH [2].In a study by Cosentino $\mathrm{C}$ et al the mean age at onset was about 70 years (range $22-90$ ). ${ }^{8}$

In the present study 4 patients $(27 \%)$ were newly detected to have Type 2 DM. In other 11 patients who had Type $2 \mathrm{DM}$, the mean duration of DM was 11.8 years (Range 7-20 years). In another study by Cosentino $\mathrm{C}$ et al diabetes was newly diagnosed in $45 \%$ of patients who presented with NKHH. Thus NKHH can be presenting manifestation of type 2 diabetes mellitus. ${ }^{9}$

The mean serum glucose levels of the patients measured in our study after the onset of NKHH was $439.93 \mathrm{mg} / \mathrm{dL}$ (range 342-532) and average $\mathrm{HbA} 1 \mathrm{c}$ was 10.98 (range 9.1-18.5). In a study by $\mathrm{Oh} \mathrm{S}$ et al, the mean serum glucose level measured after the onset of chorea was $481.5 \mathrm{mg} / \mathrm{dl}$ (ranging from 169 to 1264), HbA1c level was $14.4 \%$ (ranging from 9.9 to 19.2 ). ${ }^{2}$

In the present study the mean duration of presentation to us after symptom onset was 6.13 days (range 2- 20 days). In $\mathrm{NKHH}$, the involuntary 
movements begin acutely and may worsen over several days. $^{8}$

The hyperkinetic movements can be classified from mild chorea to severe ballism based on the type of movement and severity. Most published reports prefer usage of term hemichorea or hemiballism as bilateral involvement is rarely encountered $(<10 \%$ of cases of NKHH). In our study, 5 patients had hemichorea and 10 patients had hemiballismus. Bilateral involvement was not seen in our study. In a study by Cosentino C et al 16 patients had hemiballismus and 10 patients $(67 \%)$ had hemichorea. ${ }^{8}$ In our study left half of the body was involved in 10 patients $(67 \%)$ and right half of the body was involved in 5 patients. Left side is more commonly involved according to published cases in literature so far . The cause for laterality is not known. However in the present study all patients were having left cerebral hemisphere as dominant hemisphere based on their handedness. It can be assumed from the above observation that it was the non-dominant hemisphere basal ganglion gets commonly affected in hyperglycemia induced hemichorea-hemiballismus.

In the present study, neuroimaging revealed involvement of striatum in 13 patients and 2 patients had normal neuroimaging. Among 13 patients of striatal involvement, putamen was involved in all patients and caudate nucleus was involved in 9 cases. The neuroimaging findings are quiet distinct in $\mathrm{NKHH}$ patients, in that they show changes involving the contralateral basal ganglia. CT scan can showed mild hyper density in 8 patients and it can also be normal in some cases. ${ }^{1}$ Brain MR imaging characteristically shows T1 hyperintensity and T2 hypointensity in the basal ganglia contralateral to the movements. T2 isointensity has been described in one-third of cases. The putamen is more frequently involved than other basal ganglia structures. ${ }^{10}$ The abnormalities generally resolve over time with eventual normalization of CT or MRI.

However in some cases, lesions may persist even after the clinical resolution of NKHH. ${ }^{8}$ In a study by Cosentino $\mathrm{C}$ et al, putamen was involved in all 20 patients and the head of the caudate nucleus was affected in less than $50 \%$ of cases. ${ }^{8}$ In a study by Oh s et al MRI showed that putamen was involved in all cases. $^{2}$

Numerous theories have been proposed to explain the neuroimaging findings. These include petechial haemorrhage, myelinolysis, acute infarct, calcium deposition, decreased synthesis of g-aminobutyric acid and acetylcholine secondary to metabolic changes, or injury secondary to hyper viscosity and vasogenic oedema. ${ }^{11}$ Histopathological analysis from the hyperintense putamen showed only a slight astrocytosis and vacuolization or a fragment of gliotic brain tissue with abundant gemistocytes without hemosiderin deposition. $^{12}$
Differential diagnosis for increased $\mathrm{T} 1$ signal within the basal ganglia include early micro calcification, excessive manganese deposition, chronic hepatic encephalopathy, total parenteral nutrition and Wilson's disease.13 However in all these metabolic conditions, basal ganglion abnormality are seen bilaterally. Hyperglycemia being a metabolic abnormality affects unilateral striatum is truly a unique feature.

In the present study the mean duration for improvement of symptoms in 13 patients was 6.3 days (range 2- 12 days). Only 2 patients had persistent NKHH even after 2 months of symptom onset. These two patients were males who had presented 15-20 days after symptom onset, had right sided involuntary movements and neuroimaging was normal. Based on these observations, prognosis appears to be poor with following factors 1) hemichorea- hemiballismus affecting right half of the body 2) delayed presentation from the symptom onset 3) normal neuroimaging findings and 4) male sex. Further studies are needed to prove these prognosticating factors owing to small sample size in the present study. Generally, NKHH is a benign disorder in which hyperkinetic movements improve with glycemic control. In a study by $\mathrm{Oh} S$ et al in 39 out of 53 patients chorea had ameliorated completely. The remaining 14 cases showed some improvement during the follow-up period. The chorea had recurred only in 7 patients. $^{2}$

\section{Conclusion}

Hemichorea-hemiballism is an uncommon hyperkinetic movement disorder. The disease shows female predilection. Elderly population with poorly controlled Type 2 diabetics are most commonly affected. Lesions were commonly noted on the Right striatum with hemichorea-hemiballismus on left half of the body. Neuroimaging findings are distinct in that they show high-attenuation changes involving the contralateral basal ganglia in CT. MR imaging of brain characteristically shows T1 hyper intensity and T2 hypo intensity in the striatum contralateral to the movements. Putamen is the most commonly involved basal ganglion structure. Generally, prognosis is good. The poor prognostic factors are delayed presentation, normal MRI brain and presence of right sided hemichorea. Further studies are needed to elucidate pathogenesis of this disease and for determining prognosticating factors.

\section{References}

1. Ozgur A, Esen K, Kaleagasi H, et al. Diabetic striatopathy in a patient with hemiballism. Emerg Radiol 2015;22:347-49.

2. Oh S, Lee K, Im J, et al. Chorea associated with nonketotichyperglycemia and hyperintensity basal ganglia lesion on T1 weighted brain MRI study: a meta-analysis of 53 cases including four present cases. J Neurol Sci 2002;200:57-62. 
3. Hawley JS, Weiner WJ. Hemiballismus: current concepts and review. Parkinsonism Relat Disord 2012;18(2):12529.

4. Bedwell SF, Some observations on hemiballismus Neurol 1960;10:619-22.

5. Guo Y, Miao YW, Ji XF, Liu X, Sun XP. Hemichorea associated with nonketonic hyperglycemia: clinical and neuroimaging features in 12 patients. Eur Neurol 2014;71:299-304.

6. Ondo WG. Hyperglycemic nonketotic states and other metabolic imbalances. Handb Clin Neurol 2011;100:28791.

7. Chang X, Hong W, Yu H, Yao Y. Chorea associated with Nonketotic hyperglycemia: A case report with atypical imaging changes. NA, ed. Med 2017;9 6(45)

8. Cosentino C, Torres L, Nuñez Y, et al.

Hemichorea/hemiballism associated with hyperglycemia: report of twenty cases. Tremor Other Hyperkinet Mov. 2016
9. Lin JJ, Lin GY, Shih C, Shen WC. Presentation of striatal hyperintensity on T1-weightedMRI in patients with hemiballism-hemichoreacaused by nonketotichyperglycemia: report of seven new cases and a review of literature. J Neurol 2001;248(9):750-55.

10. Postuma RB, Lang AE. Hemiballism: revisiting a classic disorder. Lancet Neurol 2003;2(11):661-68.

11. Shan DE, Ho DM, Chang C, Pan HC, TengMM. Hemichorea-hemiballism: an explanation for MR signal changes. AJNR Am J Neuroradiol 1998;19(5):863-870.

12. Ohara S, Nakagawa S, Tabata K, Hashimoto T (2001) Hemiballism with hyperglycemia and striatal T1-MRI hyperintensity: an autopsy report. Mov Disord 2001;16:521-25.

13. Yu F, Steven A, Birnbaum L, et al. T2*-based MR imaging of hyperglycemia-induced hemichoreahemiballism. J Neurorad 2016;44(1):24-30. 